\title{
Siegesbeckia Orientalis L. Extract Attenuates Postoperative Cognitive Dysfunction, Systemic Inflammation, and Neuroinflammation
}

\author{
John Man Tak Chu ${ }^{2 \dagger}$, Wei Xiong ${ }^{1,2 \dagger}$, Ke Gang Linghu ${ }^{1}$, Yan Liu ${ }^{2}$, Yan Zhang ${ }^{2}$, \\ Guan Ding Zhao ${ }^{1}$, Michael G. Irwin ${ }^{2}$, Gordon Tin Chun Wong ${ }^{2 *}$ and Hua Yu ${ }^{1,3,4 *}$ \\ ${ }^{1}$ Institute of Chinese Medical Sciences, State Key Laboratory of Quality Research in Chinese Medicine, University of Macau, \\ Macao SAR 999078, China, ${ }^{2}$ Department of Anaesthesiology, LKS Faculty of Medicine, The University of Hong Kong, \\ Pokfulam 999077, Hong Kong, China, ${ }^{3}$ HKBU Shenzhen Research Center, Shenzhen 518000, Guangdong, China, ${ }^{4}$ School of \\ Chinese Medicine, Hong Kong Baptist University, Kowloon Tong 999077, Hong Kong, China
}

A proportion of patients experience acute or even prolonged cognitive impairment after surgery, a condition known as postoperative cognitive dysfunction (POCD). It is characterized by impairment in different cognitive domains and neuroinflammation has been implicated as one of the inciting factors as strategies targeting inflammation tend to improve cognitive performance. Siegesbeckia Orientails L. (S. Orientails) is a common Chinese medicinal herb used for managing chronic inflammatory diseases. We investigated if pretreatment with $S$. Orientails before surgery confers any neuroprotective effects in postoperative animals in terms of reducing inflammation and mitigating cognitive impairment. Three-month-old male C57BL/6N mice were fed different doses of $S$. Orientails extract for 14 days before they underwent a laparotomy. After cognitive testing they were sacrificed on postoperative day (POD) 3. Our results showed that animals with extract pretreatment demonstrated memory improvement in a dose-dependent manner compared with control. Further, evidence for the attenuation of systemic and neuroinflammation was found in the pretreated animals, along with the inhibition of inflammatory pathways and significantly reduced tau phosphorylation in the hippocampus. Taken together, these results demonstrated a neuroprotective effect of $S$. Orientails in postoperative animals, indicating a therapeutic potential of $S$. Orientails in minimizing POCD and the possibility of utilizing this traditional Chinese medicine perioperatively.

Key words: Surgery, Cognitive dysfunction, inflammation, Tau

\section{INTRODUCTION}

Received September 11,2018, Revised November 27,2018,

Accepted December 3, 2018

* To whom correspondence should be addressed.

Hua Yu, TEL: 853-8822 8540, FAX: 853-28841358

e-mail: bcalecyu@umac.mo

Gordon Tin Chun Wong, TEL: 852-22554527, FAX: 852-28551654

e-mail:gordon@hku.hk

"These authors share equal contributions in this manuscript.
Postoperative cognitive dysfunction (POCD) is a clinical condition that is characterized by impairment in multiple cognitive domains including memory, concentration, language comprehension and learning difficulties [1]. Although acute and mild cognitive decline commonly occurs after surgery, some patients develop more severe and enduring forms of cognitive dysfunction that maybe associated with increased mortality [2]. The underlying
Copyright ( $\odot$ Experimental Neurobiology 2018. www.enjournal.org
This is an Open Access article distributed under the terms of the Creative Commons Attribution Non-Commercial License (http://creativecommons.org/licenses/by-nc/4.0) which permits unrestricted non-commercial use, distribution, and reproduction in any medium, provided the original work is properly cited. 
mechanism of POCD is still unclear but postoperative neuroinflammation has been shown to be involved with the development of this condition [3]. Previous studies have demonstrated that both surgical trauma and anesthesia could initiate systemic and neuroinflammation in postoperative models [3]. For instance, the general anesthetic agent isoflurane up-regulates pro-inflammatory cytokines brain of mice [4]. Macrophages penetrate the brain and trigger glia activation and up-regulates inflammatory cytokines in the hippocampus after surgery $[5,6]$. This was associated with cognitive impairment but the degree of dysfunction was reduced by inhibiting macrophages activation and neuroinflammation in the brain [5]. Neuroinflammation also plays a critical role in synaptic dysfunction and tauopathy, in which tau hyperphosphorylation is observed under neuroinflammatory states [7]. Abnormal tau phosphorylation and accumulation are shown to interfere with synaptic function and results in cognitive impairment [7]. Collectively, these data highlight the pivotal role of neuroinflammation in the pathogenesis of POCD and the therapeutic potential of anti-inflammatory strategies to minimize POCD by inhibiting inflammation. These strategies include pharmacological pretreatment that renders an organism more resistant to a subsequent significant insult, a concept that is similar to the practice of Traditional Chinese Medicine (TCM) that emphasizes the "preventive measure".

Siegesbeckiae Herba (SH, also called Xixiancao in Chinese) is a traditional Chinese medicine (TCM) commonly used for eliminating symptoms including wind-damp, limbs weakness and detoxification as first recorded in the Newly Compiled Materia
Medica. Being one of the main plant sources of SH, the dried aerial part of Siegesbeckia Orientails L. (S. Orientails) is usually used for management of chronic inflammatory diseases such as rheumatoid arthritis (RA). In the last few decades, numerous studies have focused on the anti-inflammatory effect of S. Orientails and/or its derivative compounds in experimental RA models, with its use improving both the scores and inflammatory markers of the animal with arthritis [8]. In view of the anti-inflammatory properties of $S$. Orientails, we hypothesized that $S$. Orientails could alleviate postoperative cognitive deficits by attenuating systemic inflammation and neuroinflammation. The purpose of current study is to explore the therapeutic potential of $S$. Orientails in postoperative animals, especially in terms of cognitive performance which is correlates with the presence of inflammation and tau modulation.

\section{MATERIALS AND METHODS}

\section{Animals}

3-month-old C57BL6/N mice were obtained from The University of Hong Kong. The handling of animal and all procedures were conducted in accordance with National Institutes of Health guide for the care and use of Laboratory animals and Animals (Control of Experiments) Ordinance, Hong Kong, China. The use of animals was approved by the Department of Health, Hong Kong and Committee on the Use of Live Animals in Teaching and Research, The University of Hong Kong. All efforts were made to minimize animal numbers and suffering. In the current report, mice were divided into 6 groups: Sham control (Ctrl), surgery (Lap), low
A

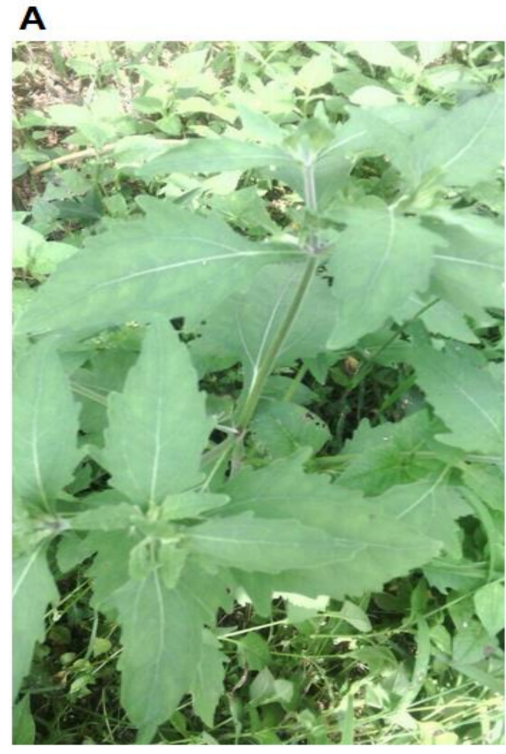

\section{B}

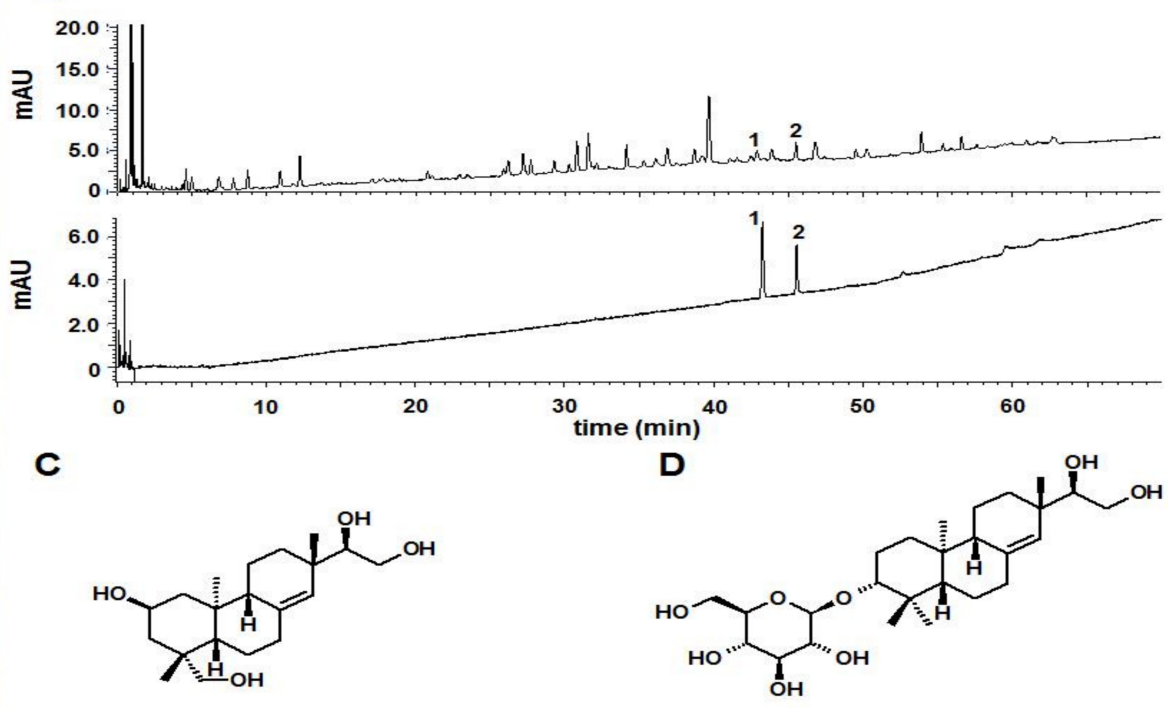

Fig. 1. Preparation and chemical profile of S. Orientails extract. (A) Pictures of S. Orientails L.. (B) Chemical profile of S. Orientails analyzed by UPLC. 1, Kerinol; 2, Darutoside. (C) Chemical structure of kerinol. (D) Chemical structure of darutoside. 
dose drug control ( $0.75 \mathrm{~g} / \mathrm{kg} /$ day), high dose drug control (1.5 g/ $\mathrm{kg} /$ day), low dose drug with surgery (0.75 g/kg/day plus Lap) and high dose drug with surgery ( $1.5 \mathrm{~g} / \mathrm{kg} /$ day plus Lap).

\section{Preparation and characterization of S. Orientails L. extract}

The herb of $S$. Orientails (Fig. 1A) was collected from Gubao Town (Xiuwen County, Guiyang, Guizhou Province, China) (time of collection: August, 2015), and authenticated by the corresponding author, Dr. Hua YU. The voucher specimens (No. SO-002) were deposited at the Institute of Chinese Medical Sciences, University of Macau, Macao, China.

For use in the experiments, a powdered SO (100 g) was refluxextracted twice with 10 -fold volume of $50 \%$ ethanol (v/w) for 1 hr for each. The combined extract was cooled, filtered, and then concentrated under reduced pressure to remove the ethanol. Subsequently, the concentrated extract was lyophilized with a Virtis Freeze Dryer (The Virtis Company, New York, USA). The powdered S. Orientails extract (yield: $15.4 \%$, brown color) was kept at $4^{\circ} \mathrm{C}$ for further experiments.

Quantifications of two major active compounds (kirenol and da- rutoside) in the $S$. Orientails extract was performed using a Waters ACQUITY-UPLC CLASS system (Waters Corp., Milford, USA) coupled with an ACQUITY UPLC HSS T3 column (150 mm×2.1 $\mathrm{mm}, 1.8 \mu \mathrm{m})$ maintained at $40^{\circ} \mathrm{C}$. Elution was performed with a mobile phase of $\mathrm{A}(0.2 \% \mathrm{H} 3 \mathrm{PO} 4$ in water $)$ and $\mathrm{B}(0.2 \% \mathrm{H} 3 \mathrm{PO} 4$ in $\mathrm{ACN}$ ) under a gradient program: $0 \sim 5 \mathrm{~min}, 17 \% \mathrm{~B} ; 5 \sim 15 \mathrm{~min}$, $17 \% \sim 25 \% \mathrm{~B} ; 15 \sim 30 \mathrm{~min}, 25 \sim 50 \%$. The flow rate was $0.4 \mathrm{ml} / \mathrm{min}$ and the injection volume was $2 \mu$. The analytes were monitored at the UV wavelength of $215 \mathrm{~nm}$. Between two injections, the column was washed with $100 \%$ B for 2 min and equilibrated with the initial mobile phase for 5 mins.

\section{Surgical and drug treatments}

To examine if $S$. Orientails extract ameliorates postoperative cognitive dysfunction, mice assigned to drug treatment groups were given the agent for 14 days prior to surgery. Timeline of whole experiment is shown in Fig. 2A. The extract was dispersed in 1X PBS and $100 \mu \mathrm{l}$ of extract solution was orally administrated to mice at the dosages of $0.75 \mathrm{~g} / \mathrm{kg} /$ day and $1.5 \mathrm{~g} / \mathrm{kg} /$ day for 14 consecutive days. Sham control and surgery groups were given equivalent

A

\begin{tabular}{|c|c|c|c|c|c|}
\hline $\begin{array}{c}\text { Drug } \\
\text { treatment } \\
\text { begin }\end{array}$ & $\begin{array}{c}\text { Drug } \\
\text { treatment } \\
\text { end }\end{array}$ & Laparotomy & $\begin{array}{l}\text { NOR } \\
\text { Day } 1\end{array}$ & $\begin{array}{l}\text { NOR } \\
\text { Day } 2\end{array}$ & $\begin{array}{c}\text { NOR } \\
\text { Day } 3\end{array}$ \\
\hline Day 1 & Day 14 & Day 15 & POD 1 & POD 2 & POD \\
\hline
\end{tabular}

$\mathbf{B}$

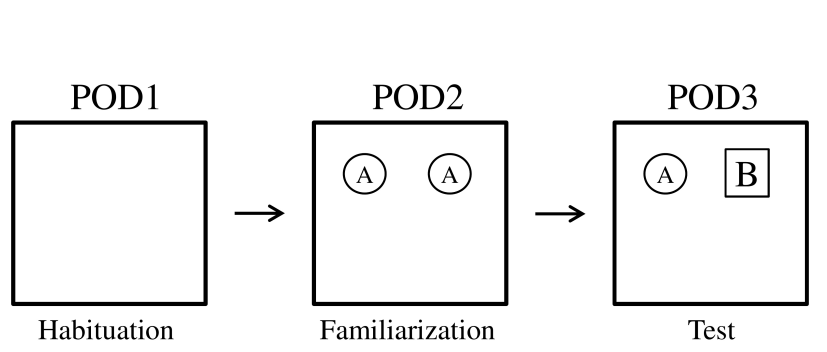

C Novel object recognition performance

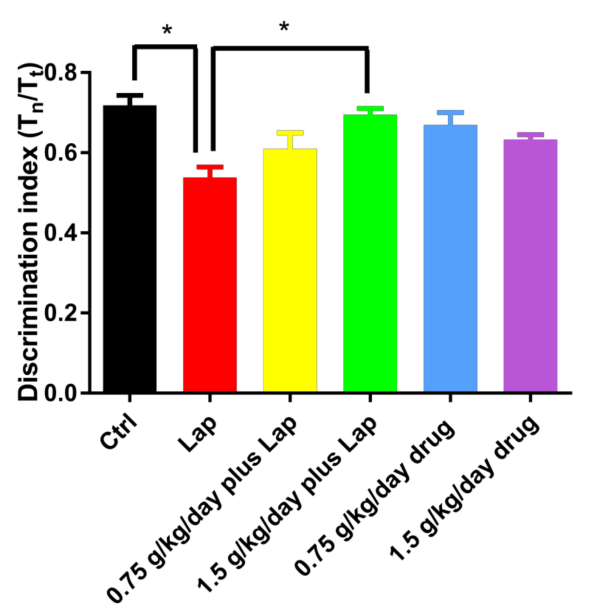

Fig. 2. S. Orientails improved hippocampal dependent memory function in postoperative animals in dose-dependent manner. The mice demonstrated impaired object recognition memory function on postoperative day 3. (A) Timeline of the drug treatment and behavioral test. (B) Schematic diagram for novel object recognition test. (C) A shorter novel object exploration time was observed in the surgical group while $S$. Orientails extract increased exploration time of the novel object. Data are presented as the mean and SEM $(n=6) .{ }^{*} p<0.05$ between groups. 
amount of 1X PBS. On day 15 the mice underwent a midline laparotomy under 3\% sevoflurane general anesthesia in 100\% oxygen. After incision, the gastrointestinal tract was exteriorized and rubbed by fingers for $1 \mathrm{~min}$ to mimic surgery, after which it was replaced into the peritoneal cavity followed by wound closure. The mice were allowed to recover before further testing.

\section{Novel object recognition test}

Hippocampal dependent object recognition memory was determined by the novel object recognition (NOR) test as described before but with modifications [9]. Briefly, on postoperative day (POD) 1 , mice were put in an open field box $(50 \times 50 \mathrm{~cm})$ with no objects for 10 mins for habituation. On POD 2, mice were placed in the same box with 2 identical objects for 10 mins for familiarizing the object. Twenty-four hours after familiarization on POD 3, one of the two objects was replaced with a novel object. The mice were allowed to stay in the box for 10 mins and the behavior of the animals was video recorded. Schematic diagram is shown in Fig. 2B. Object exploration was scored by the amount of time with the nose pointed towards and located within $2 \mathrm{~cm}$ of the object. The discrimination index was calculated by the formula: $T_{n} / T_{t}$, where $T_{n}$ is the time of exploring novel object while $T_{t}$ is the total time of exploring novel and familiarized object. After completing the novel object recognition test, the mice were sacrificed by $\mathrm{CO}_{2}$ asphyxiation. $1 \mathrm{ml}$ of blood was extracted by cardiac puncture and serum was isolated after 1,300 g centrifugation for 10 mins. Liver and hippocampal tissues were then dissected out from animals for subsequent real time PCR and Western Blot experiments.

\section{mRNA extraction and real time PCR}

Hepatic and hippocampal mRNA were isolated by RNAiso plus (Takara, Japan). Tissues were first homogenized in RNAiso plus and vigorously mixed with chloroform. After $10000 \mathrm{~g}$ centrifugation at $4^{\circ} \mathrm{C}$, the upper aqueous layer was isolated. mRNA pellets were precipitated by isopropanol and washed with 75\% ethanol. It was then dissolved in diethyl pyrocarbonate (DEPC) treated water. $1 \mu \mathrm{g}$ of mRNA was converted to complementary DNA sequence by reversed transcription using cDNA synthesis kit according to manufactures' protocol (Takara, Japan). Levels of cDNA of different inflammatory cytokines were assessed by real time PCR with respective primers: 1$)$ IL- $1 \beta$, forward: CCTCCTTGCCTCTGATGG, reverse: AGTGCTGCCTAATGTCCC; 2) IL-6, forward: TTCACAAGTCCGGAGAGGAG, reverse: TCCACGATTTCCCAGAGAAC; 3) IL-8, forward: TGCCGTGACCTCAAGATGTGCC, reverse: CATCCACAAGCGTGCTGTAGGTG; 4) TNF- $\alpha$, forward: CCCCAGTCTGTATCCTTCT, reverse: ACTGTCCCAGCATCTTGT; 5) GAPDH, forward:
ATTCAACGGCACAGTCAA, reverse: CTCGCTCCTGGAAGATGG.

\section{Measurement of serum inflammatory markers}

Inflammatory cytokine expression in the serum was evaluated by MILLIPLEX MAP mouse cytokine/chemokine magnetic bead panels (EMD Millipore Corp., Billerica, MA). All primary data points were collect on a Luminex MAGPIX system. Protein samples and detection substrates were incubated on the plate with specific antibodies-conjugated magnetic beads coated on each well. Fluorescence signal detection and analysis were performed according to the manufacturer's protocol. Concentrations of IL$1 \beta$, IL-6, MIP-2 (homologues of IL- 8 ) and TNF- $\alpha$ in serum were determined.

\section{Western blot}

Hippocampal tissue was dissected out and homogenized as mentioned above. Proteins were extracted with RIPA lysis buffer (Cellsignal, Danvers, MA) supplemented with protease and phosphatase inhibitors (Roche, Berlin, German). Protein samples were quantified by BCA protein assay. Proteins were resolved by SDSPAGE gel and transferred to PVDF membrane. After blocking with $2 \%$ non-fat milk, membranes were probed overnight at $4^{\circ} \mathrm{C}$ with different primary antibodies (JNK, p-JNK, p65 and p-p65 were from Cellsignal, MA; P-tau S396 and $\beta$-actin were from Thermo Fisher Scientific, MA; Pan-tau was from DAKO, Japan), followed by respective HRP-conjugated secondary antibodies for $1 \mathrm{hr}$. Protein bands were visualized by enhanced chemiluminescence (ECL) reagents and signals were captured by X-ray film. The intensities of protein bands were quantified by Image J analysis software.

\section{Statistical analysis}

Comparison between different groups of treatment was analyzed by one-way ANOVA with Turkey post-hoc test. Significant differences were considered between groups when $\mathrm{p}<0.05$.

\section{RESULTS}

\section{Characterization of S. Orientails extract}

Kerinol (Fig. 1C) and darutoside (Fig. 1D) are the two most important compounds in $S$. Orientails, both demonstrating antiinflammatory effects in vitro and in vivo $[10,11]$. Moreover, kerinol is the recommended chemical marker use for quality control of the $S$. Orientails herb by the Chinese Pharmacopeia. Therefore, quantifications of kerinol and darutoside in S. Orientails extract were performed. As illustrated in Fig. 1B, kerinol and darutoside 
can be chromatographically separated using the developed UPLC method. The contents of kerinol and darutoside in the in S. Orientails extract were determined to be $0.24 \pm 0.01 \%$ and $1.47 \pm 0.11 \%$, respectively.

\section{S. Orientails extract ameliorated cognitive impairment in postsurgical animals in a dose dependent manner}

Memory deficits on POD 3 were observed in the surgical group as manifested by a significant reduction of novel object exploration time (Fig. 2). Drug treatment alone did not exert any adverse effects on memory function. In the surgery plus drug groups, although no significant improvement of cognitive deficit was found with the low dose $(0.75 \mathrm{~g} / \mathrm{kg} /$ day $)$ treatment, the high dose $(1.5 \mathrm{~g} / \mathrm{kg} /$ day $)$ treatment significantly improved memory function as reflected by the increase in the discrimination index (Fig. 2, $\mathrm{p}=0.0265$ between Ctrl and Lap; $\mathrm{p}=0.0376$ between Lap and $1.5 \mathrm{~g} /$ $\mathrm{kg} /$ day plus Lap). No significant differences were found between other groups; $\mathrm{p}>0.05)$. These data demonstrated that pretreatment with the extract improved postoperative memory function in dose dependent manner.

\section{S. Orientails pretreatment improved both systemic and neuroinflammation in postoperative animals}

To investigate if extract pretreatment could attenuate inflammatory responses in postoperative animals, mRNA of pro-inflammatory cytokines in the liver were examined by real time PCR. Significant up-regulation of IL-6 was observed in the laparotomy group while high dose extract pretreatment ameliorated the increased mRNA expression of these pro-inflammatory cytokines (Fig. 3A) ( $\mathrm{p}=0.0117$ between Ctrl and Lap; $\mathrm{p}=0.0127$ between Lap and 1.5 $\mathrm{g} / \mathrm{kg} /$ day plus Lap, non-significance was found between other groups; $\mathrm{p}>0.05$ ). In addition, cytokine levels in the serum were examined by Milliplex assay. In line with the results from real time PCR, serum concentration of IL-6 ( $\mathrm{p}=0.015$ between Ctrl and Lap;

A

mRNA in liver

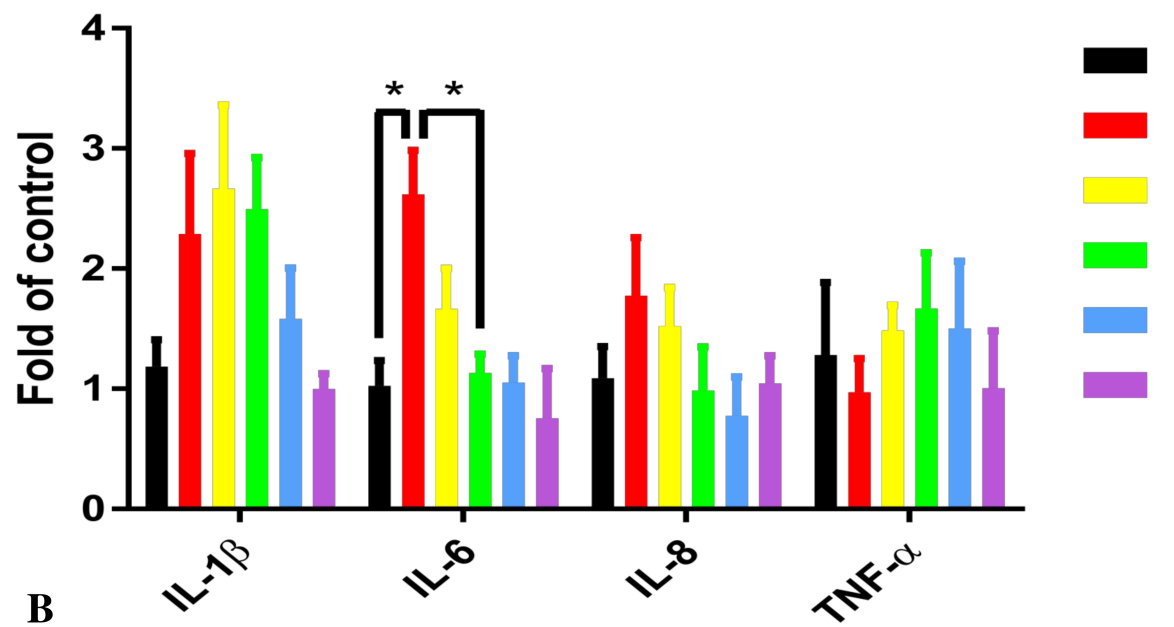

Ctrl

Lap

0.75 g/kg/day plus Lap

$1.5 \mathrm{~g} / \mathrm{kg} /$ day plus Lap

$0.75 \mathrm{~g} / \mathrm{kg} /$ day drug

$1.5 \mathrm{~g} / \mathrm{kg} /$ day drug
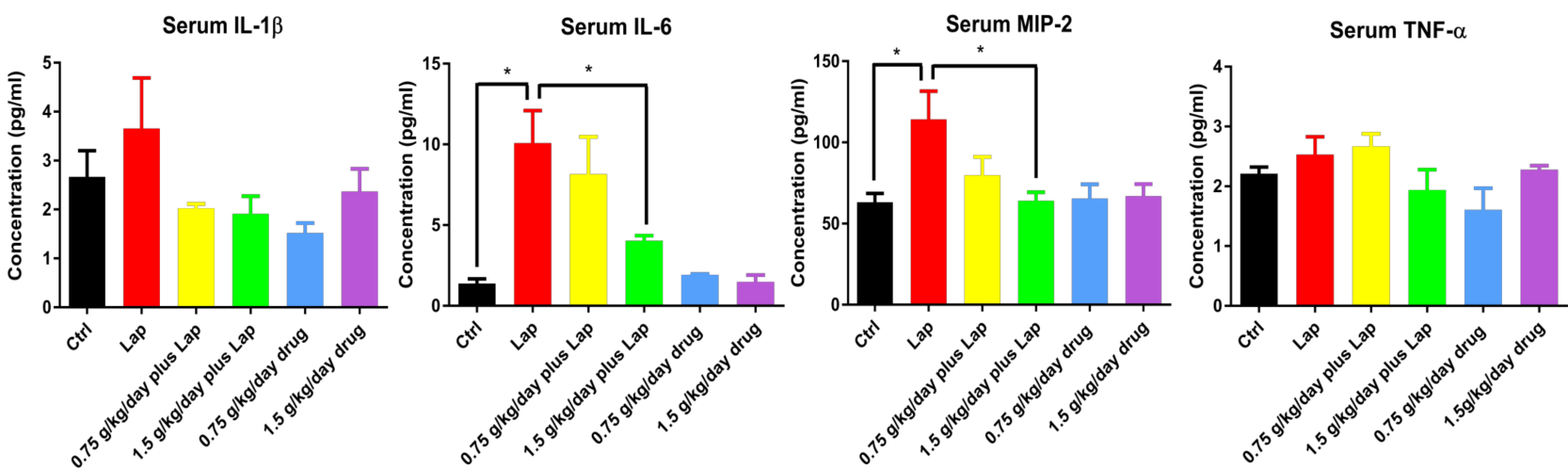

Fig. 3. S. Orientails inhibited surgery induced systemic inflammation. Laparotomy increased pro-inflammatory cytokine gene expressions (A) in the liver and (B) level of inflammatory cytokines in serum. Postoperative mice received extract pretreatment inhibited the systemic inflammatory responses. Data correspond to the mean and SEM $(n=4-5)$. GAPDH was used as mRNA internal control. ${ }^{*} \mathrm{p}<0.05$ between groups. 
$\mathrm{p}=0.044$ between Lap and $1.5 \mathrm{~g} / \mathrm{kg} /$ day plus Lap, non-significance was found between other groups; $\mathrm{p}>0.05$ ) and MIP-2, the homologue of IL-8, were significantly increased in surgery group while reduced after high dose extract treatment (Fig. 3B. $\mathrm{p}=0.0137$ between Ctrl and Lap; $\mathrm{p}=0.0236$ between Lap and $1.5 \mathrm{~g} / \mathrm{kg} /$ day plus Lap, No significant difference was found between other groups; $\mathrm{p}>0.05$ ) Finally, to examine the neuroinflammatory response, mRNA expression of inflammatory cytokines were examined by real-time PCR. Increase in IL- $1 \beta$ ( $p=0.0337$ between Ctrl and Lap; $\mathrm{p}=0.0416$ between Lap and $1.5 \mathrm{~g} / \mathrm{kg} /$ day plus Lap, no significant difference between other groups; $p>0.05)$ and IL-6 ( $p=0.0339$ be- tween Ctrl and Lap; $\mathrm{p}=0.0482$ between Lap and $1.5 \mathrm{~g} / \mathrm{kg} /$ day plus Lap, no significant difference between other groups; $p>0.05$ ) were observed in the hippocampi of the surgical group. These increases were attenuated by high dose extract (Fig. 4). Overall, these results demonstrated that the extract reduced inflammation in both peripherally and centrally.

\section{Pro-inflammatory JNK and NF- $\mathrm{JB}$ pathways were inhib- ited by S. Orientails extract}

After observing the inflammatory response in the hippocampus, we further examined if extract pretreatment inhibited neuroin-

\section{mRNA in brain}
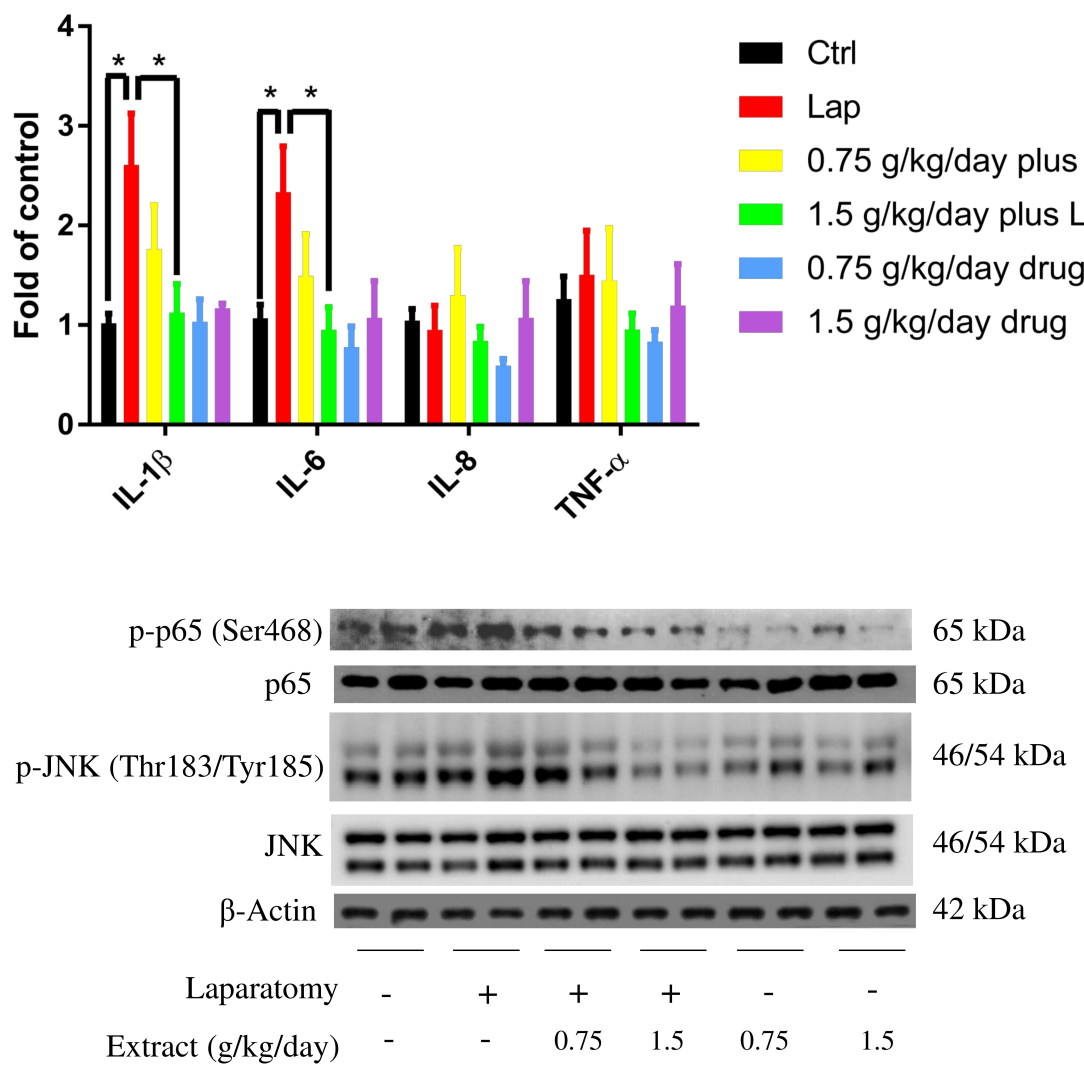

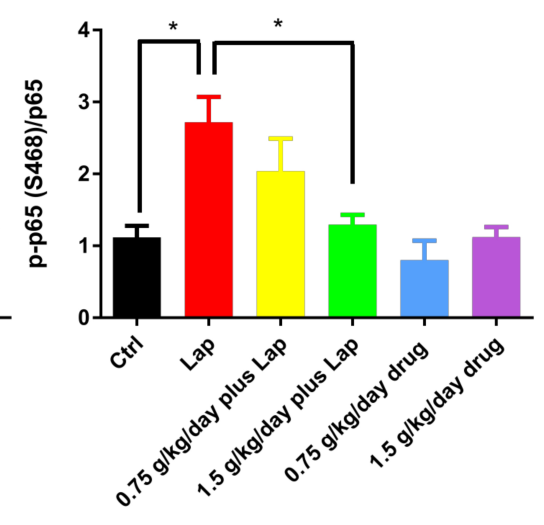

Fig. 4. S. Orientails reduced neuroinflammation in the hippocampus induced by surgery. Neuroinflammatory response was evaluated in the hippocampus by examining inflammatory cytokines gene expression. Data correspond to the mean and SEM $(\mathrm{n}=4-5)$. GAPDH was used as mRNA internal control. ${ }^{*} \mathrm{p}<0.05$ between groups.
Fig. 5. S. Orientails inhibited activation of JNK and NF- $\kappa$ B pathways in the hippocampus in postoperative animals. Activation of JNK and NF- $\kappa \mathrm{B}$ pathways in the hippocampus by laparotomy while pretreatment of $S$. Orientails extract inhibited the activities of both pathways. Representative blots against phosphorylated JNK and p65 in the hippocampus of different groups of animals. Graphs below showing quantification of western blot. Data correspond to the mean and SEM $(n=4)$ and represents the band densities that were normalized with endogenous JNK and p 65 respectively. ${ }^{*} \mathrm{p}<0.05$ between groups. 
flammation through modulating pro-inflammatory pathways (JNK and NF-kB) by measuring phosphorylation of JNK and p65 subunit using Western blot. In postsurgical animals, significant upregulation of phosphorylated JNK ( $\mathrm{p}=0.0226$ between Ctrl and Lap; $\mathrm{p}=0.0403$ between Lap and $1.5 \mathrm{~g} / \mathrm{kg} /$ day plus Lap, non-significance was found between other groups; $\mathrm{p}>0.05)$ and $\mathrm{p} 65$ ( $\mathrm{p}=0.0182$ between Ctrl and Lap; $\mathrm{p}=0.021$ between Lap and $1.5 \mathrm{~g} / \mathrm{kg} /$ day plus Lap, non-significance was found between other groups; $p>0.05$ ) was observed in the hippocampus, whereas extract pretreatment reduced the phosphorylation of both molecules in a dose dependent manner (Fig. 5). These results imply that extract pretreatment may attenuate neuroinflammatory responses through suppressing JNK and p65 activities and subsequent nucleus translocation in the hippocampus.

\section{Tau phosphorylation in the hippocampus was reduced af- ter $S$. Orientails extract pretreatment}

Neuroinflammatory response is closely related to tau phosphorylation, which leads to the destabilization of tau from microtubules and subsequent memory deficits [7]. To examine if surgery induces tau phosphorylation in the hippocampus and whether extract pretreatment impedes this process, protein samples were subjected to Western blotting and examined by phosphorylated tau antibody at Serine 396 epitome. Significant up-regulation of
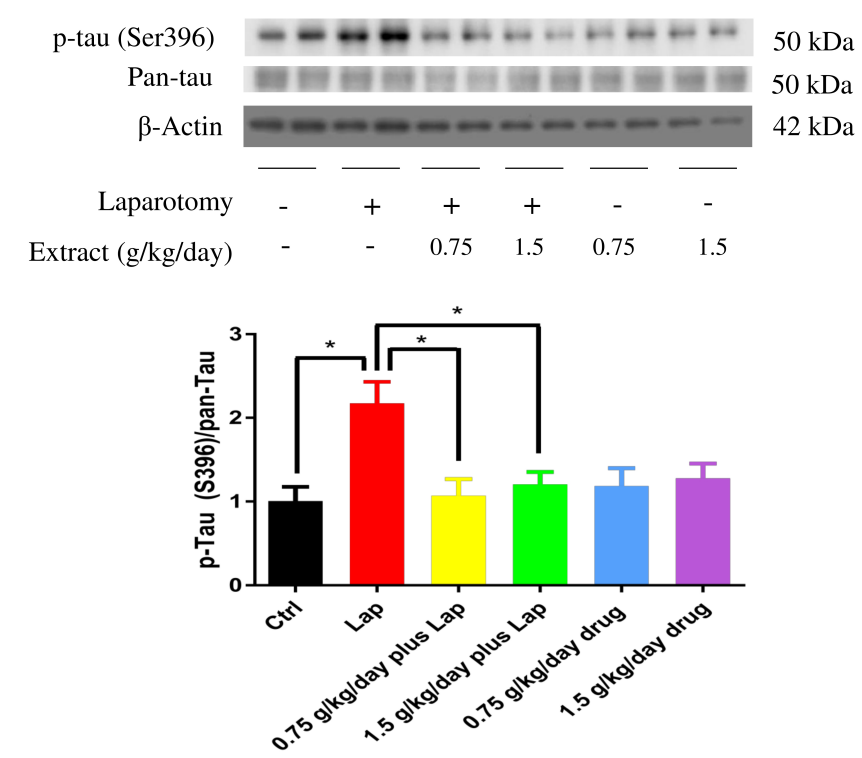

Fig. 6. S. Orientails reduced phosphorylation of tau in the hippocampus induced by surgery. Increased tau phosphorylation was observed in the hippocampus of postoperative mice while $S$. Orientails extract pretreatment reduced tau phosphorylation. Graphs below showing quantification of Western blot. Data correspond to the mean and SEM ( $n=4)$ and represents the band densities that were normalized with total tau (pan-tau). ${ }^{*} \mathrm{p}<0.05$ between groups. tau phosphorylation was found in the hippocampus after surgery, while extract pretreatment reduced tau phosphorylation (Fig. 6) ( $\mathrm{p}=0.0159$ between Ctrl and Lap; $\mathrm{p}=0.0268$ between Lap and 1.5 $\mathrm{g} / \mathrm{kg} /$ day plus Lap, non-significance was found between other groups; $\mathrm{p}>0.05$ ). These results indicated that extract may provide a neuroprotective effect to postoperative animals through reducing neuroinflammation and tau phosphorylation.

\section{DISCUSSION}

Substantial evidence suggest that surgery induces cognitive dysfunction in patients in whom a continuous inflammatory response is present [3]. Apart from various physical barriers, perioperative inflammation was once considered as the second line of defense mechanism to protect human from pathogens after surgery. Nevertheless, it is increasingly recognized that several postoperative complications are related to inflammation including atrial fibrillation [12], hemorrhage [13] and cognitive dysfunction [14]. Various therapeutic strategies targeting inflammation throughout the surgical period have been suggested to combat these complications. In agreement with previous reports, our results suggest that surgery induces systemic and neuroinflammatory response in postoperative animals [3]. Furthermore, activation of pro-inflammatory pathways was observed, which was associated with an increase in tau phosphorylation and cognitive impairment, thus showing that treatment using $S$. Orientails extract before surgery partially prevented these pathological changes and demonstrated the therapeutic potential of $S$. Orientails in preventing POCD.

It is well established that peripheral inflammation could trigger a neuroinflammatory response in the brain [15]. Chronic neuroinflammation has been shown to accompany deterioration in cognitive function. For instance, inflammation cytokines induce the hyperactivation of astrcotyes [16], which may trigger the release of other cytokines or gliotransmitter such as GABA that affect neuronal activity and plasticity in the AD model [17]. Inhibiting neuroinflammation in turn resulted in amelioration of astrogliosis and improved subsequent cognitive impairment in $\mathrm{AD}$ [18]. On the other hand, therapeutic strategies targeting systemic inflammation may improve neuroinflammation and ameliorate neuronal damage, synaptic dysfunction and cognitive performance in different models, including POCD [19]. Traditional Chinese medicine has been used over many centuries and mounting evidence has demonstrated anti-inflammatory properties. S. Orientails has been widely used in treating arthritis through inhibiting systemic inflammation [20,21]. From previous studies, it was shown to improve paw edema in experimental RA animal model, accompanied with the reduction of IL-6 in serum [22]. 
From in vitro assay, $S$. Orientails was shown to reduce IL-6, TNF- $\alpha$ and NO in RAW264.7 cells. Topical application of S. Orientails active components reduces inflammatory cytokines in carrageenan and complete Freund's adjuvant induced inflammatory models [23]. These data demonstrated that $S$. Orientails is a potent natural anti-inflammatory herb which may exert broad range of beneficial effects by ameliorating inflammatory responses.

In view of these anti-inflammatory properties, we examined whether extract from $S$. Orientails can improve the cognitive function in postoperative animal. We have shown a significant increase in the novel object exploration time in postoperative animals with 14 days of extract pretreatment compared with surgery only group, implying an improvement in hippocampal dependent recognition memory. Based on this observation, we examined if the extract could reduce systemic inflammation and neuroinflammation in postoperative mice. Along with better preservation of cognitive function, there was a reduction of pro-inflammatory cytokines IL-6 and IL-8 in the liver and serum of postoperative animals with extract pretreatment in a dose dependent manner (Fig. 3). At the same time, extract pretreatment reduced the mRNA of IL- $1 \beta$ and IL-6 in the hippocampus (Fig. 4). These results demonstrated that extract pretreatment reduced inflammatory responses by suppressing pro-inflammatory cytokines in both the periphery and in the hippocampus, a result similar to that found in experimental RA models.

To further elucidate how the extract could reduce the production of pro-inflammatory cytokines in brain, we investigated if the activation of pro-inflammatory intracellular signaling was modulated. $\mathrm{JNK}$ and $\mathrm{NF}-\kappa \mathrm{B}$ are major pathways involve in inflammation $[24,25]$ and they respond to external stimulus such as reactive oxygen species, bacteria or virus. In neurodegeneration, activation of JNK and NF- $\kappa B$ are observed in the brains of Alzheimer's Disease models $[26,27]$. Both molecules are phosphorylated and translocated to the nucleus, binding to the promoter and enhancing the transcriptional activities of the genes, thus increasing the synthesis of pro-inflammatory cytokine [24, 25]. Over-activation of JNK and NF- $\kappa B$ in the brain are shown to worsen inflammatory responses while inhibition of these molecules ameliorates neuroinflammation and subsequent cognitive dysfunction. Our results demonstrated that surgery leads to the phosphorylation of both JNK and NF- $\kappa B$ in the brain, which was in line with the increase in pro-inflammatory cytokine gene expression. In contrast, extract pretreatment significantly reduced the phosphorylation of both JNK and NF- $\kappa B$ (Fig. 4), thus further confirming that the extract reduces the neuroinflammatory response by impeding the activation of pro-inflammatory pathways in the hippocampus.

One of the possible explanations for the anti-inflammation effect of $S$. Orientails is the modulation of peripheral immune activities. Previous reports have shown that surgery-induced neuroinflammation was partially triggered by the activation and penetration of peripheral immune cells into the central nervous system [28]. These cells could activate inflammatory pathways and stimulate the production of inflammatory cytokines in the brain. On the other hand, previous studies also indicated that $S$. Orientails could modulate the activities of peripheral immune cells [29,30], which implies the that $S$. Orientails may also inhibit surgery-induced neuroinflammation through regulating peripheral immunity. However, apart from interlukins, we could not observe significant modulation of other cytokines such as TNF- $\alpha$ in the brain. It may be accounted by the differential expression time of respective cytokines. For example, from in vitro studies, up-regulation of TNF- $\alpha$ mRNA was observed in lipopolysaccharide (LPS) challenged bone marrow derived dendritic cells as early as $1 \mathrm{hr}$ and returning to basal levels within 24 hrs [31], which may explain the failure of observing TNF- $\alpha$ modulation in our current study. It needs further investigation to confirm if other cytokine expression will be modulated at earlier time points in the postoperative period.

We further asked how the attenuation of neuroinflammation by the extract may modulate tau phosphorylation in the hippocampus. Tau is an important microtubule associated protein in which abnormal phosphorylation and aggregation of tau protein is a pathological hallmark in cognitive dysfunction model [7]. Upon phosphorylation, tau was shown to be dissociated from microtubule which undergo aggregation and oligomerization and exert neurotoxicity or inhibit the function of synapse, leading to synaptic and neuronal dysfunction. It has been shown that inflammatory cytokines such as IL-6 could induce tau phosphorylation which deteriorate neuronal degeneration [32]. In our model, tau phosphorylation was up-regulated in the hippocampus after surgery. With extract pretreatment, reduction of phosphorylated tau was observed compared with surgery alone group (Fig. 5). These results imply that $S$. Orientails extract may have beneficial effect on postoperative animals through anti-inflammation and a reduction in the tau phosphorylation burden in the hippocampus, which is consistent with the previous findings that reducing tau phosphorylation improved neuronal and synaptic deficit in neurodegeneration such as Alzheimer's disease [33]. For future studies, other tau phosphorylation sites and the aggregation of tau protein in the hippocampus maybe required to give a full spectrum of how $S$. Orientails can modulate tauopathy in postoperative animals.

Despite the promising effect of $S$. Orientails in the current study, some issues would still need to be addressed. For example, pulmonary toxicity was reported for the water extract of Herba Siegesbeckiae (HS) in mice [34], and the toxic compounds were 
identified to be presented mainly in the aqueous-soluble part of the extract [35]. The median lethal dose (LD50) for oral acute toxicity of the water extract of HS in mice was reported to be $146.7 \mathrm{~g}$ (herb) $/ \mathrm{kg}$, and the toxic effects was observed when the dosage was higher than $24.4 \mathrm{~g}(\mathrm{herb}) / \mathrm{kg}$ in a 2-week sub-acute test. Moreover, the acute toxicity of the 70\% ethanol extracts of HS (LD50: 267.00 $\mathrm{g}(\mathrm{herb}) / \mathrm{kg}$ ) was determined to be lower than that of the water extract (LD50: $147.91 \mathrm{~g}$ (herb) $/ \mathrm{kg}$ ). Although the maximum dosage of S. Orientails was set at $10 \mathrm{~g}$ (herb)/kg in this study, which was much lower than the LD50 of the acute and sub-acute dosages of the water extract (LD50: $24.4 \mathrm{~g}(\mathrm{herb}) / \mathrm{kg}$ ), the potential toxicity of the $S$. Orientails should be carefully considered and further investigation is needed in future.

To conclude, this study presented in vivo data on the neuroprotective effect of $S$. Orientails extract against surgery induced cognitive dysfunction. With pretreatment using higher doses of $S$. Orientails extract, surgery induced systemic and neuroinflammation were ameliorated, as shown by the inhibition of inflammatory pathway JNK and NF-kB and the reduction of interlukin inflammatory cytokines expression respectively. This is associated with the attenuation of tauopathy in the hippocampus that may underlie the neuroprotective mechanism in our model. Taken together, we have demonstrated that $S$. Orientails may have beneficial effect in postoperative subjects.

\section{ACKNOWLEDGEMENTS}

This work was supported by HKU Seed Fund for Basic Research (201511159317 and 201611159054), National Natural Science Foundation of China (NSFC, No. 81470170), the Research Committee of the University of Macau (SRG2015-00060-ICMSQRCM, MYRG2017-00178-ICMS, MYRG2018-00043-ICMS), Guangzhou Science and Technology Innovation and Development of Special Funds (EF007/ICMS-YH/2018/GSTIC) and the opening fund of the State Key Laboratory of Quality Research in Chinese Medicine of University of Macau (No. SKL-QRCM-2014-2016).

\section{REFERENCES}

1. Deiner S, Silverstein JH (2009) Postoperative delirium and cognitive dysfunction. Br J Anaesth 103 Suppl 1:i41-i46.

2. Steinmetz J, Christensen KB, Lund T, Lohse N, Rasmussen LS; ISPOCD Group (2009) Long-term consequences of postoperative cognitive dysfunction. Anesthesiology 110:548555.

3. Hovens IB, Schoemaker RG, van der Zee EA, Absalom AR,
Heineman E, van Leeuwen BL (2014) Postoperative cognitive dysfunction: involvement of neuroinflammation and neuronal functioning. Brain Behav Immun 38:202-210.

4. Wu X, Lu Y, Dong Y, Zhang G, Zhang Y, Xu Z, Culley DJ, Crosby G, Marcantonio ER, Tanzi RE, Xie Z (2012) The inhalation anesthetic isoflurane increases levels of proinflammatory TNF- $\alpha$, IL-6, and IL-1 $\beta$. Neurobiol Aging 33:1364-1378.

5. Degos V, Vacas S, Han Z, van Rooijen N, Gressens P, Su H, Young WL, Maze M (2013) Depletion of bone marrowderived macrophages perturbs the innate immune response to surgery and reduces postoperative memory dysfunction. Anesthesiology 118:527-536.

6. Feng X, Valdearcos M, Uchida Y, Lutrin D, Maze M, Koliwad SK (2017) Microglia mediate postoperative hippocampal inflammation and cognitive decline in mice. JCI Insight 2:e91229.

7. Metcalfe MJ, Figueiredo-Pereira ME (2010) Relationship between tau pathology and neuroinflammation in Alzheimer's disease. Mt Sinai J Med 77:50-58.

8. Nguyen TD, Thuong PT, Hwang IH, Hoang TK, Nguyen MK, Nguyen HA, Na M (2017) Anti-hyperuricemic, antiinflammatory and analgesic effects of Siegesbeckia orientalis L. Resulting from the fraction with high phenolic content. BMC Complement Altern Med 17:191.

9. Bevins RA, Besheer J (2006) Object recognition in rats and mice: a one-trial non-matching-to-sample learning task to study 'recognition memory'. Nat Protoc 1:1306-1311.

10. Son PT, Giang PM, Taylor WC (2005) NMR studies of darutoside, a rare ENT-pimarane glucoside. Nat Prod Res 19:503507.

11. Lu Y, Xiao J, Wu ZW, Wang ZM, Hu J, Fu HZ, Chen YY, Qian RQ (2012) Kirenol exerts a potent anti-arthritic effect in collagen-induced arthritis by modifying the T cells balance. Phytomedicine 19:882-889.

12. Boos CJ, Anderson RA, Lip GY (2006) Is atrial fibrillation an inflammatory disorder? Eur Heart J 27:136-149.

13. Cai B, Deitch EA, Ulloa L (2010) Novel insights for systemic inflammation in sepsis and hemorrhage. Mediators Inflamm 2010:642462.

14. Perry VH (2004) The influence of systemic inflammation on inflammation in the brain: implications for chronic neurodegenerative disease. Brain Behav Immun 18:407-413.

15. Perry VH, Cunningham C, Holmes C (2007) Systemic infections and inflammation affect chronic neurodegeneration. Nat Rev Immunol 7:161-167.

16. Balasingam V, Tejada-Berges T, Wright E, Bouckova R, Yong VW (1994) Reactive astrogliosis in the neonatal mouse brain 
and its modulation by cytokines. J Neurosci 14:846-856.

17. Jo S, Yarishkin O, Hwang YJ, Chun YE, Park M, Woo DH, Bae JY, Kim T, Lee J, Chun H, Park HJ, Lee DY, Hong J, Kim HY, Oh SJ, Park SJ, Lee H, Yoon BE, Kim Y, Jeong Y, Shim I, Bae YC, Cho J, Kowall NW, Ryu H, Hwang E, Kim D, Lee CJ (2014) GABA from reactive astrocytes impairs memory in mouse models of Alzheimer's disease. Nat Med 20:886-896.

18. Avila-Muñoz E, Arias C (2014) When astrocytes become harmful: functional and inflammatory responses that contribute to Alzheimer's disease. Ageing Res Rev 18:29-40.

19. Huang C, Irwin MG, Wong GT, Chang RC (2018) Evidence of the impact of systemic inflammation on neuroinflammation from a non-bacterial endotoxin animal model. J Neuroinflammation 15:147.

20. Hong YH, Weng LW, Chang CC, Hsu HF, Wang CP, Wang SW, Houng JY (2014) Anti-inflammatory effects of Siegesbeckia orientalis ethanol extract in in vitro and in vivo models. BioMed Res Int 2014:329712.

21. Nguyen TD, Thuong PT, Hwang IH, Hoang TK, Nguyen MK, Nguyen HA, Na M (2017) Anti-hyperuricemic, antiinflammatory and analgesic effects of Siegesbeckia orientalis L. Resulting from the fraction with high phenolic content. BMC Complement Altern Med 17:191.

22. Hong YH, Weng LW, Chang CC, Hsu HF, Wang CP, Wang SW, Houng JY (2014) Anti-inflammatory effects of Siegesbeckia orientalis ethanol extract in in vitro and in vivo models. BioMed Res Int 2014:329712.

23. Wang JP, Zhou YM, Ye YJ, Shang XM, Cai YL, Xiong CM, Wu YX, Xu HX (2011) Topical anti-inflammatory and analgesic activity of kirenol isolated from Siegesbeckia orientalis. J Ethnopharmacol 137:1089-1094.

24. Tak PP, Firestein GS (2001) NF-kappaB: a key role in inflammatory diseases. J Clin Invest 107:7-11.

25. Ip YT, Davis RJ (1998) Signal transduction by the c-Jun Nterminal kinase (JNK)--from inflammation to development.
Curr Opin Cell Biol 10:205-219.

26. Okazawa H, Estus S (2002) The JNK/c-Jun cascade and Alzheimer's disease. Am J Alzheimers Dis Other Demen 17:7988.

27. O’Neill LA, Kaltschmidt C (1997) NF-kappa B: a crucial transcription factor for glial and neuronal cell function. Trends Neurosci 20:252-258.

28. Degos V, Vacas S, Han Z, van Rooijen N, Gressens P, Su H, Young WL, Maze M (2013) Depletion of bone marrowderived macrophages perturbs the innate immune response to surgery and reduces postoperative memory dysfunction. Anesthesiology 118:527-536.

29. Sun HX, Wang H (2006) Immunosuppressive activity of the ethanol extract of Siegesbeckia orientalis on the immune responses to ovalbumin in mice. Chem Biodivers 3:754-761.

30. Shao NQ, Zhao M, Wang HQ, Zhu XX (2012) Experimental research of effect of herba siegesbeckiae on immunological function of mice. Chin Arch Tradit Chin Med 8:66.

31. Gais P, Tiedje C, Altmayr F, Gaestel M, Weighardt H, Holzmann B (2010) TRIF signaling stimulates translation of TNF-a mRNA via prolonged activation of MK2. J Immunol 184:5842-5848.

32. Quintanilla RA, Orellana DI, González-Billault C, Maccioni RB (2004) Interleukin-6 induces Alzheimer-type phosphorylation of tau protein by deregulating the $\mathrm{cdk} 5 / \mathrm{p} 35$ pathway. Exp Cell Res 295:245-257.

33. Giacobini E, Gold G (2013) Alzheimer disease therapy-moving from amyloid- $\beta$ to tau. Nat Rev Neurol 9:677-686.

34. Guan JH, Xue Z, Liu BC, Ren JB (2008) An experimental study on pulmonary toxicity water extracts of Siegesbeckia pubescens on mice. Zhongguo Zhongyao Zazhi 33:28202822.

35. Jianhong G, Xiangping P, Bingcheng L (2009) Toxic effect of different extracts of herba siegesbeckiae on mice. J Shanxi Coll Tradit Chin Med 10:15. 